\title{
IR CHARACTERISTICS OF TYPE II CEPHEIDS IN THE LMC AND IN THE GALAXY
}

\author{
C. DAVID LANEY \\ South African Astronomical Observatory \\ P.O. Box 9 \\ Observatory 7935 \\ Cape \\ South Africa
}

\begin{abstract}
JHK observations have been obtained of Type II Cepheids in the LMC, in globular clusters, and in the galactic field. The P-L relations at $\mathrm{J}$ and $\mathrm{H}$ imply an LMC distance modulus consistent with Mv 0.6 for RR Lyrae stars. Modest excesses at $\mathrm{K}$ are seen in some LMC objects. One previously reported LMC Type II Cepheid appears to be a Type I Cepheid, and two others are much redder in J-H than normal Type II Cepheids. HV11211 is a Type I Cepheid in the SMC.
\end{abstract}

Previous reported JHK data for Type II LMC Cepheids is limited to the nine single-phase observations by Welch (1987). In the present paper, comparisons are made between preliminary JHK results for 19 alleged LMC Type II Cepheids (an average of four observations per star), 25 globular cluster Cepheids and 13 Type II Cepheids in the field $\left(\mathrm{b}>10^{\circ}\right)$. All observations were made at SAAO and have been reduced to the standard system of Carter (1990).

In the two-colour diagram (Fig 1), there are three stars with very red $(\mathrm{J}-\mathrm{H})_{0}$ for their periods (and at most a modest excess at K), HV5756, HV12631, and M12V1. All are also excessively bright in the combined P-L diagram (Fig 2). Even RV Tauri stars at minimum light (Wing 1986) normally have spectral types corresponding to much bluer $(\mathrm{J}-\mathrm{H})_{0}$.

Four LMC Type II Cepheids (including three previously reported by Welch), have (H$\mathrm{K})_{0}>0.4$, and five more have redder $(\mathrm{H}-\mathrm{K})_{0}$ than any galactic Type II Cepheid in this sample except V1711 Sgr. As noted by Welch, the presence of an excess at K may be related to RV Tauri characteristics, but none of the nine RV Tauri stars (mostly RVC) in this sample shows a conspicuous excess. It is possible that the LMC Type II Cepheids of long period are more similar to galactic RVA or RVB stars, where excesses at K are common (Lloyd Evans 1985). The intrinsic JHK colours of the galactic variables in the present sample (except V1711 Sgr) are close to those of Type I Cepheids (Fig 1) and of nearby unreddened giants and dwarfs.

Two long-period LMC stars, HV12625 and HV925, are anomalously faint in the P-L diagram (Fig 2). Their colours seem normal, and there is nothing in the photographic light curves (Gaposchkin 1970) to suggest that the periods might be halved. Stars with longer periods than HV12625 have therefore been excluded from the calculation of a distance modulus for the LMC. Using the remaining stars one obtains $18.42+/-0.13(\mathrm{~J})$, and $18.44+/-0.13(\mathrm{H})$, assuming that $\mathrm{M}_{\mathrm{V}}$ (horizontal branch) for globular clusters is 0.6 . This is in good agreement with previous results using the same assumption (Feast 1988). 
110

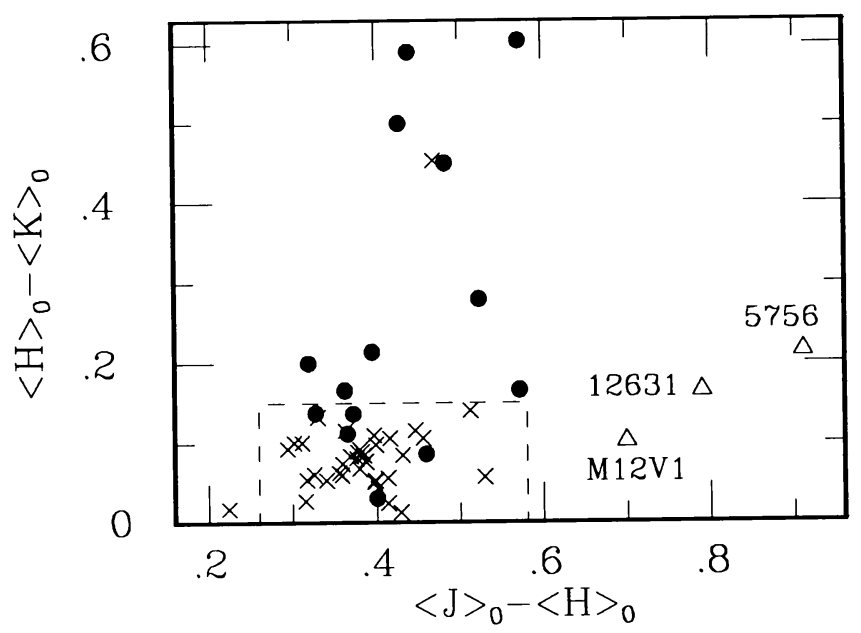

Figure 1. Combined IR 2-colour diagram for LMC (circles) and galactic (crosses) Type II Cepheids, and objects probably not normal Type II Cepheids (triangles). LMC Type I Cepheid observations fall inside the dashed box.

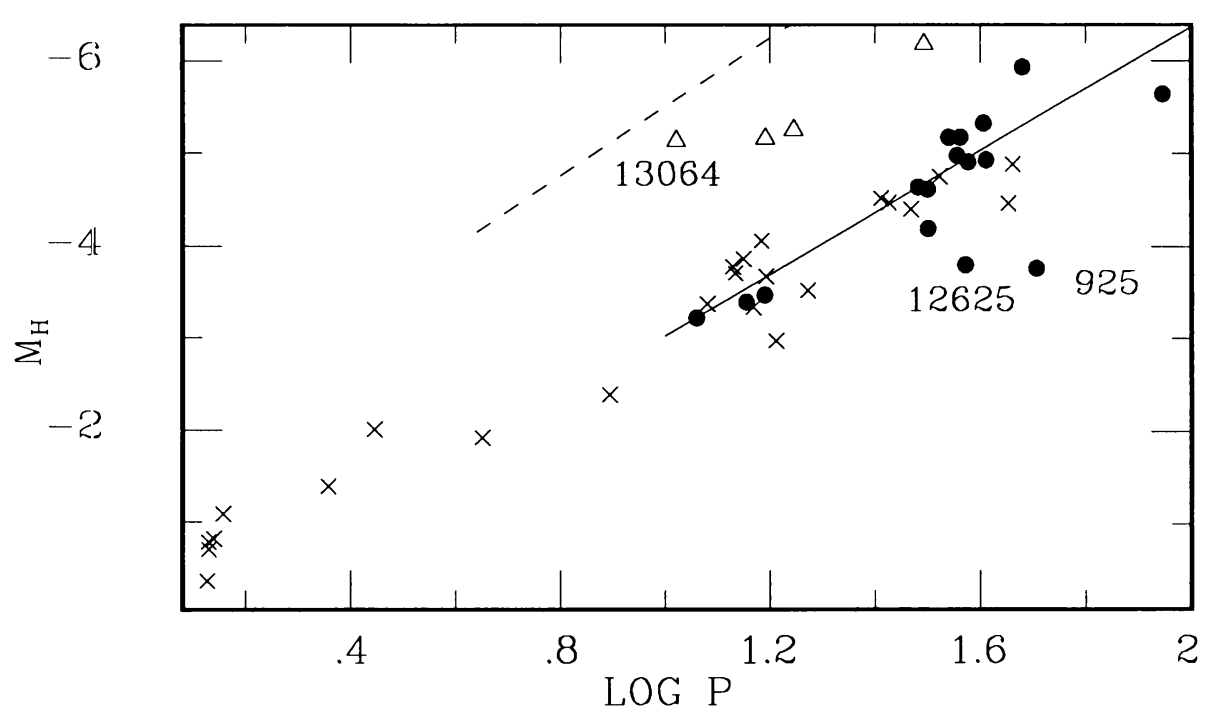

Figure 2. Combined P-L diagram in H. The dashed and solid lines are P-L relations for Type I and Type II Cepheids. Symbols as in Figure 1. 
HV13064 has been suggested as a probable Type II Cepheid (Connolly 1975). The JHK data indicate that it is a highly reddened Type I Cepheid. The Type I status of HV11211, important because of its position at the extreme near end of the SMC wing, has been questioned recently (Welch et al. 1985). With the aid of additional unpublished data, I have calculated the Fourier parameter $R_{21}$ (Simon and Lee 1981) for J, and the ratio of the $J$ and $J-K$ amplitudes. Comparison to similar ratios for Galactic Type I and II Cepheids (Laney and Stobie, and Laney, both in preparation) indicates that HV11211 is a Type I Cepheid and hence a member of the SMC.

\section{References}

Carter, B.X., (1990), Mon. Not. R. astr. Soc. 242,1.

Connolly, L.P., (1975), PhD. thesis, Univ. of Arizona.

Feast, M.W., (1988), The Extragalactic Distance Scale, p.9, eds. S. van den Bergh \& C.J. Pritchet Ast. Soc. Pacific, San Francisco.

Gaposchkin, S., (1970), Smithsonian Astr. Obs. Special Rep., no. 310.

Lloyd Evans, T., (1985), Mon. Not. R. astr. Soc. 217, 493.

Simon, N.R. \& Lee, A.S., (1981), Astrophys. J. 248, 291.

Welch, D.L., (1987), Ap. J. 317, 672.

Welch, D.L., McAlary, C.W., Madore, B.F., McLaren, R.A., (1987), Ap. J. 321, 162.

Wing, R.F., (1986), J. Amer. Assoc. Var. Star Obs. 317, 672 